\title{
Dimensionamiento en rotura a flexión de secciones de hormigón armado. Un planteamiento compacto
}

\section{Strength design of reinforced concrete sections in flexion. A compact approach}

L.M. Gil-Martín ${ }^{(*)}$, D. López-Martín ${ }^{(*)}$, E. Hernández-Montes ${ }^{(*)}$, M. A. Aschheim ${ }^{(*)}$

RESUMEN

Este artículo es una revisión general del procedimiento tradicional de dimensionamiento en rotura de secciones de hormigón armado. El procedimiento que recogen la mayor parte de los libros de texto actuales data de los años 50 del siglo XX y es susceptible de ser simplificado. En este artículo el planteamiento se reformula y se presenta de una manera más compacta, lo que supone un cambio tanto desde el punto de vista docente como desde el punto de vista profesional del hormigón armado. El Teorema de Armado a Flexión confirma que las ingeniosas soluciones propuestas por Whitney o Wuczkowsky, corresponden a los mínimos de un problema general que puede ser planteado de una forma más racional. El hecho de presentar el problema de forma compacta, empleando los diagramas RSD permite poder elegir soluciones alternativas de armado de menor impacto medioambiental.

$451-16$

Palabras clave: Dimensionamento en rotura; diseño de hormigón armado; diseño de hormigón pretensado.

\section{SUMMARY}

This paper is an overview of the traditional method of strength design of reinforced concrete sections. The procedure, presented in the current textbooks, dates from the 50's of XX century and it can be simplified. In this work the approach is reformulated and presented in a more compact way, which implies a change both from the educational point of view and from the professional point of view of reinforced concrete. Theorem of Optimal Reinforcement of RC Sections confirms that the ingenious solutions proposed by Whitney and Wuczkowsky correspond to the minimum of a general problem that can be developed more rationally. Presenting the problem in compact form, using diagrams RSD allowed to choose alternative solutions of reinforcement with reduced environmental impact.

Keywords: Strenght design; reinforced concrete design; prestressed concrete design.

\footnotetext{
(*) Universidad de Granada, (España).

(**) University of Santa Clara, (EEUU). 
1. Tratamiento tradicional para el diseño en rotura de elementos de hormigón armado sometidos a flexión.

2. Distribución de deformaciones según la hipótesis de las secciones planas.

\section{INTRODUCCIÓN}

El diseño en rotura de secciones de hormigón armado es un tema muy estudiado que ha ido evolucionando a lo largo de la historia del hormigón. Prácticamente la totalidad de los textos de hormigón consideran la división representada en el diagrama de flujo de la Figura 1, en la que aparecen métodos ingeniosos tales como la clasificación de Whitney (1) entre pequeñas y grandes excentricidades (que aún se sigue empleando hoy en día). Para grandes excentricidades aún hoy se sigue empleando el Teorema de Ehlers (2) conocido también como método de Wuczkowsky (3) en la literatura francesa, aunque su aplicación no siempre conduce a buenos resultados, según demostró Páez (4).

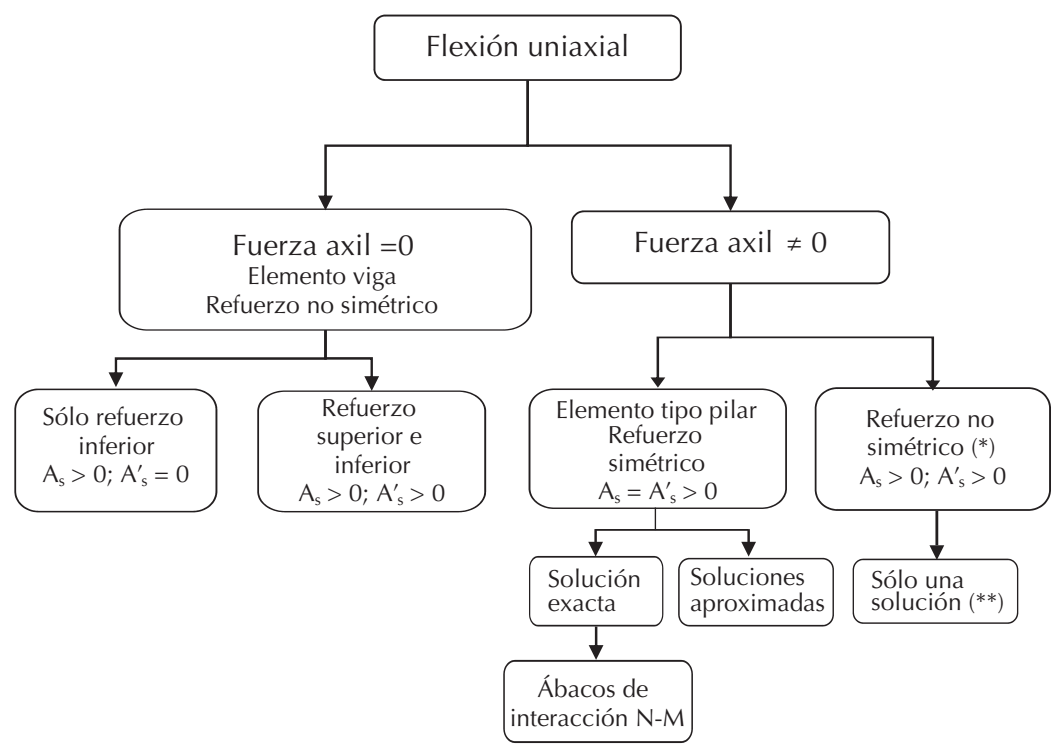

(*) Método de Whitney: división entre grandes y pequeñas excentricidades

$\left.{ }^{* *}\right)$ Método de Wuczkowsky (conocido también como Teorema de Ehlers)

En la práctica, la manera tradicional de plantear el diseño en rotura de elementos de hormigón sometidos a flexión (Figura 1), ha resultado muy útil puesto que evita la necesidad de resolver un problema matemático que generalmente es no lineal, con funciones escalonadas -i.e. definidas a trozos- $y$ que, como se verá, tiene infinitas soluciones.

A lo largo de la historia de la Teoría de Estructuras y de la Resistencia de Materiales ingenios similares a los anteriores han pasado a la historia debido al avance de las mejoras matemáticas (Cremona, método de Cross...). De hecho, prestigiosos ingenieros como Nathan Newmark dedicaron años al desarrollo de métodos gráficos hoy desaparecidos.

En esta publicación se aborda desde un punto de vista analítico el diseño en rotura de secciones de hormigón armado con objeto de permitir un diseño más compacto que pueda reemplazar a los métodos tradicionales. Además se analiza la manera en que este nuevo tipo de desarrollo puede afectar a los profesionales de la ingeniería y de la docencia del cálculo de hormigón.

\section{PLANOS DE DEFORMACIÓN Y DE ROTURA}

\subsection{La hipótesis de las secciones planas (HSP) o hipótesis de Bernoulli}

La teoría de la viga es la que se deriva de la hipótesis de las secciones planas. En ingeniería se diferencia entre la teoría de la viga de Bernoulli - cuando no se incluye el efecto de la deformación por cortante por ser despreciable frente a las deformaciones ocasionadas por el momento flector- y la teoría de la viga de Timoshenko -cuando la deformación por cortante sí es tenida en cuenta-.

La ventaja de aceptar la HSP es que conocidas las deformaciones unitarias de dos puntos de la sección transversal (p.ej. $\varepsilon_{\text {sup }}$ y $\varepsilon_{\text {inf }}$ ver Figura 2) es posible obtener la deformación unitaria de cualquier fibra de la sección transversal. Generalmente las dos variables que definen la deformación unitaria de la sección transversal son la deformación a nivel del centro de gravedad de la sección y la curvatura (i.e. $\varepsilon_{\text {cdg }}$ y $\varphi$, ver Figura 2). De esta forma la deformación de cualquier fibra de la sección transversal, corresponda a hormigón o a acero, situada a una distancia y del c.d.g. (ver Figura 2)

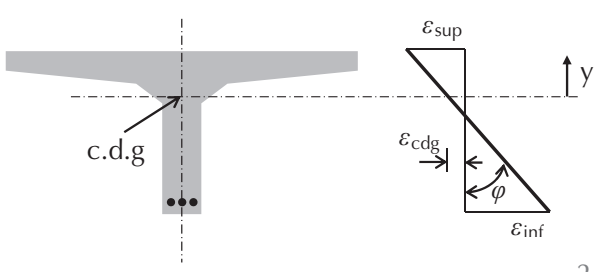

vendrá dada por: [1]

[1]

$$
\varepsilon\left(y, \varepsilon_{c d g}, \varphi\right)=\varepsilon_{c d g}+\varphi y
$$

En el caso de que la fibra cuya deformación se pretende estimar corresponda a acero de pretensado, será necesario considerar además una predeformación inicial $\varepsilon_{\mathrm{p} 0}$ : [2]

[2] $\varepsilon_{p}\left(y, \varepsilon_{c d g}, \varphi, \varepsilon_{p 0}\right)=\varepsilon_{c d g}+\varphi y-\varepsilon_{p 0}$

En la formulación anterior se ha considerado deformación positiva la de compresión y curvatura positiva la que produce tracciones en la parte inferior de la sección transversal.

\subsection{Tratamiento de la rotura en las normativas EC2 y ACI318}

La rotura de una sección es un estado límite último asociado al fallo de la sección por 
agotamiento del hormigón a compresión o, bajo algunas hipótesis, del acero a tracción.

El EC2 define una serie de posibles planos de deformaciones unitarias que corresponden a planos de rotura (Figura 3a) para los cuales el acero agota a tracción (planos que pivotan en torno al punto A de la Figura 3a) o el hormigón agota a compresión (planos que pivotan en torno al punto $\mathrm{B}$ o $\mathrm{C}$ de la Figura 3a).

El modelo de hormigón del Eurocódigo 2 considera que la deformación última de agotamiento del hormigón a compresión es diferente en situación de flexo-compresión que en compresión pura. Debido a esto aparece el plano de agotamiento de deformación constate $\varepsilon_{\mathrm{c} 2} \circ \varepsilon_{\mathrm{c} 3}$ (según se considere el modelo parábola-rectángulo o el modelo rectangular del hormigón, respectivamente). Punto $\mathrm{C}$ de la Figura 3 a.

La norma $\mathrm{ACl}-318$ es más sencilla y considera que la rotura se produce únicamente por agotamiento del hormigón a compresión $\left(\varepsilon_{c \max }=0.003\right)$. Debido a esta hipótesis todos los planos de rotura pasan por un único pivote (punto B de la Figura 3b).

En el caso de que se considere para el acero un modelo bilineal sin endurecimiento, el Eurocódigo 2 permite, como la norma $\mathrm{ACl}$ 318, no limitar la deformación del acero a tracción. En este caso el pivote A de la Figura 3a desaparecería.

Si se observan los planos de rotura representados en la Figura 3 se puede apreciar que la deformación en rotura de toda la sección transversal se puede definir a partir de una sola variable: la profundidad de la fibra neutra $(x)$. El dominio de $x$, en el caso de la norma ACl-318 y del EC2 -cuando no se considera el pivote $A$ - es $\mathrm{x} \in[0, \infty)$ mientras que en el caso del EC2 -cuando se considera el pivote $A$ - es $x \in(-\infty, \infty)$.

Si se consideran los planos de rotura definidos por el EC2, sin limitar la deformación del acero, la deformación de la sección transversal a nivel de cualquier fibra situada a $\xi$ del extremo superior se puede obtener a partir de [3] (ver Figura 4):

$$
\begin{aligned}
& \varepsilon(\xi, x)=\left\{\begin{array}{llr}
\varepsilon_{\mathrm{cu} 3} \frac{x-\xi}{x} & \text { si } & 0 \leq x<h \\
\varepsilon_{\mathrm{c} 3} \frac{x-\xi}{x-\Xi} & \text { si } & x \geq h
\end{array}\right. \\
& \text { donde } \Xi=h\left(1-\frac{\varepsilon_{\mathrm{c} 3}}{\varepsilon_{\mathrm{cu} 3}}\right)
\end{aligned}
$$

En el caso de que la deformación del acero a tracción se limite, lo que equivale a considerar el pivote $\mathrm{A}$, la función $\varepsilon(\xi, x)$ estaría a) Deformaciones de rotura según el Euro-código 2

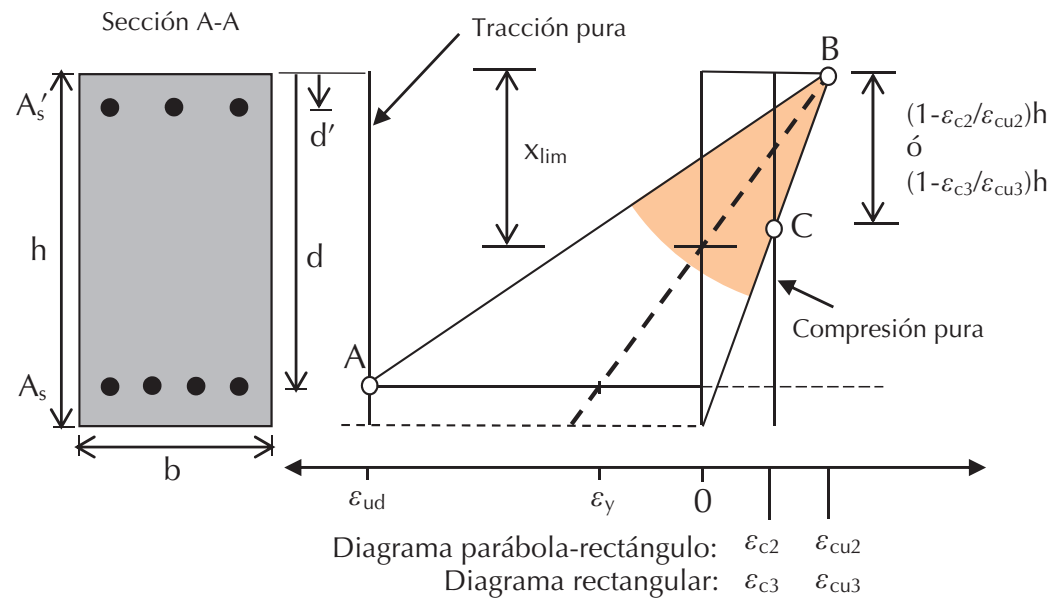

A Deformación última del acero en tracción

B Deformación última del hormigón en compresión con flexión

C Deformación última del hormigón en compresión pura

b) Deformaciones de rotura según la $\mathrm{ACl}-318-08$

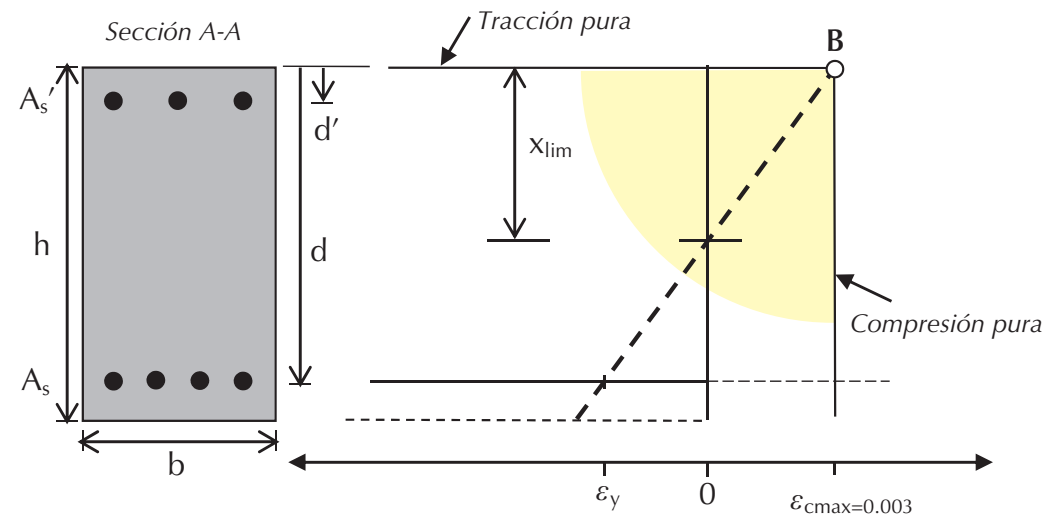

B Deformación última del hormigón en compresión con flexión definida en tres dominios en lugar de en dos, como sucede en la ecuación [3].

Si se consideran los planos de deformación de la ACl-318 la ecuación [3] estaría definida en sólo un dominio. Sin embargo, esta
3. Posibles distribuciones de deformaciones unitarias en estado límite último

a) Eurocódigo 2 y b) ACl-318-08

4. Plano de deformación de rotura definido por la variable $\mathrm{x}$.

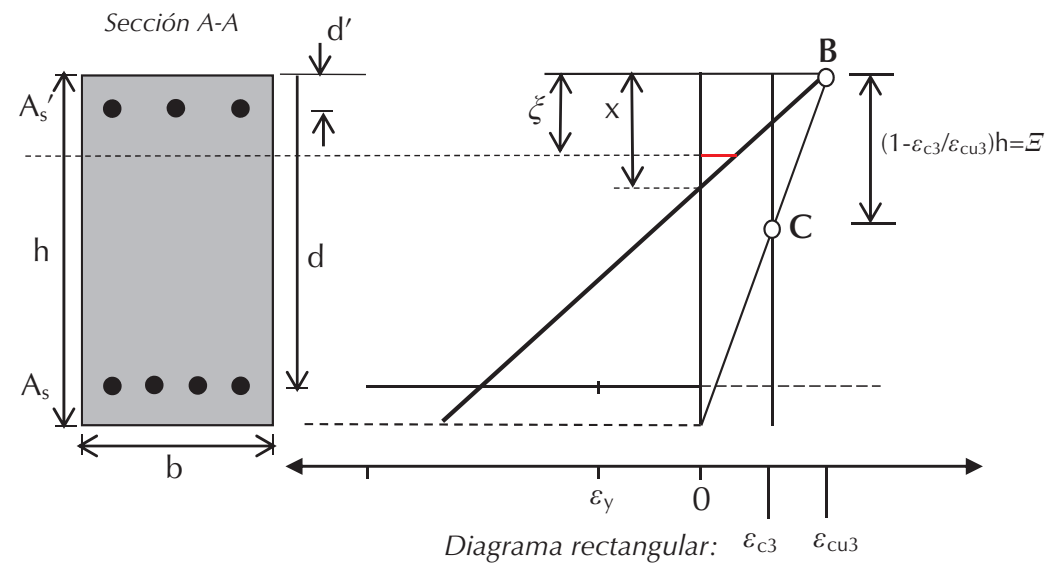

B Deformación última del hormigón en compresión con flexión C Deformación última del hormigón en compresión pura 
norma introduce un coeficiente de seguridad $\Phi$ que afecta a la ecuación [3] y que está definido en tres dominios en función de $x$, lo que elimina la simplicidad de la ecuación [3] bajo la hipótesis de la ACl-318.

\section{LOS MODELOS DE LOS MATERIALES LA COMPOSICIÓN DE FUNCIONES Y EL EQUILIBRIO}

\subsection{El modelo de hormigón en rotura.}

El hormigón es un material cuya respuesta tensión-deformación $\left(\sigma_{c}-\varepsilon_{c}\right)$, es no lineal y varía, entre otros factores, con la edad del hormigón y con la duración de la carga. Dada la importancia del diseño en rotura se han ideado unos modelos sencillos aplicables exclusivamente a esta situación. El Eurocódigo 2 recoge tres modelos para el hormigón: el rectangular, el parábola-rectángulo y el bilineal.

Si se considera el modelo rectangular, también conocido como bloque de tensiones equivalente, la resultante del volumen de compresiones $N_{c}$ puede expresarse, en el caso de secciones rectangulares, en función de la profundidad de la fibra neutra, $x$, como: [4]

$$
\mathrm{N}_{\mathrm{c}}(x)=\left\{\begin{array}{ccc}
\eta f_{\mathrm{cd}} \lambda b x & \text { si } & 0 \leq x<\frac{h}{\lambda} \\
\eta f_{\mathrm{cd}} \lambda h & \text { si } & x \geq \frac{h}{\lambda}
\end{array}\right.
$$

donde $f_{c d}$ es el valor de la resistencia de diseño del hormigón; $h$ y $b$ son el canto y el ancho de la sección transversal, respectivamente; $\lambda$ y $\eta$ son parámetros que dependen de la resistencia del hormigón y que se definen en el EC2.

Mientras que el diagrama rectangular se expresa generalmente en función únicamente de $x$, el modelo parábola-rectángulo del hormigón se presenta como una función escalonada en función de la deformación unitaria del hormigón a compresión $\left(\sigma_{\mathrm{c}}\left(\varepsilon_{\mathrm{c}}\right)\right.$, ver §3.1.7 del Eurocódigo 2).

\subsection{El modelo de acero}

El modelo de acero que se emplea en el cálculo en rotura de secciones transversales de hormigón es el bilineal, generalmente sin endurecimiento, y simétrico (es decir, se considera la misma expresión a tracción y a compresión. Gil-Martín et ál. (5) demostraron que para las separaciones máximas entre cercos permitidas por las actuales normativas esta hipótesis es correcta incluso cuando el posible pandeo de las barras es considerado).
Por simplicidad, en las ecuaciones [4] no se ha considerado la presencia de la armadura embebida en la sección transversal de hormigón. Para tener esto en cuenta se corregirá el modelo de acero de la siguiente manera: [5]

$\sigma_{s}(\varepsilon)=\left\{\begin{array}{ccc}f_{y d}-\eta f_{c d} & \text { si } & \varepsilon \geq \frac{f_{y d}-\eta f_{c d}}{E_{s}} \\ E_{s} \varepsilon & \text { si }-\frac{f_{y d}}{E_{s}}<\varepsilon<\frac{f_{y d}-\eta f_{c d}}{E_{s}} \\ -f_{y d} & \text { si } & \varepsilon \leq-\frac{f_{y d}}{E_{s}}\end{array}\right.$

\subsection{Expresiones de las tensiones del acero en función de $x$}

Como se ha comentado anteriormente, la deformación en rotura queda perfectamente definida a partir de una sola variable: la profundidad de la fibra neutra $(x)$.

En el caso frecuente de una sección transversal con dos niveles de armadura $A_{s}^{\prime}$ y $A_{s}$ situados a $d^{\prime}$ y $d$ de la fibra superior, respectivamente; la deformación y la tensión en rotura a nivel de las armaduras se pueden obtener a partir de las ecuaciones [3] y [5]: [6]

$$
\begin{aligned}
& \varepsilon_{s}^{\prime}(x)=\varepsilon\left(d^{\prime}, x\right) \\
& \varepsilon_{s}(x)=\varepsilon(d, x) \\
& \sigma_{s}^{\prime}(x)=\sigma_{s}\left(\varepsilon_{s}^{\prime}(x)\right)=\left(\sigma_{s} \mathrm{O} \varepsilon_{s}^{\prime}\right)(x) \\
& \sigma_{s}(x)=\sigma_{s}\left(\varepsilon_{s}(x)\right)=\left(\sigma_{s} \mathrm{O} \varepsilon_{s}\right)(x)
\end{aligned}
$$

donde " $"$ " simboliza la composición de dos funciones matemáticas.

Estas expresiones pueden ser extendidas a cualquier posición de la armadura, dado que ésta viene definida, con carácter general, por la coordenada $\xi$ de la expresión [3].

\subsection{El equilibrio}

El estado de tensiones existente en cualquier sección transversal debe equilibrar a los esfuerzos que la solicitan $\left(N_{d^{\prime}} M_{d}\right)$.

En el caso de flexión compuesta en ELU, el axil y el momento engendrados por las tensiones internas vienen dados por: [7]

[7]

$$
\begin{aligned}
N_{u}(x)= & N_{c}(x)+A_{s}^{\prime} \sigma_{s}^{\prime}(x)+A_{s} \sigma_{s}(x) \\
M_{u}(x)= & N_{c}(x)\left(\frac{h}{2}-z_{c}(x)\right)+A_{s}^{\prime} \sigma_{s}^{\prime}(x)\left(\frac{h}{2}-d^{\prime}\right)+ \\
& +A_{s} \sigma_{s}(x)\left(\frac{h}{2}-d\right)
\end{aligned}
$$


Siendo $z_{c}$ el braco mecánico de la resultante del volumen de compresiones respecto a la fibra superior, definido como: [8]

$$
z_{c}(x)=\left\{\begin{array}{llc}
\frac{\lambda x}{2} & \text { si } & 0 \leq x<\frac{h}{\lambda} \\
\frac{h}{2} & \text { si } & x \geq \frac{h}{\lambda}
\end{array}\right.
$$

En la ecuación [7] se podía haber tenido un carácter más general si se sustituye el término $h / 2$ por el $y_{\text {cdg }}$ de la sección bruta. El término $h / 2$ es para secciones rectangulares. La misma generalización puede hacerse en la ecuación [4], sustituyendo $b$ por $b(x)$.

\section{EL PROBLEMA DE COMPROBACIÓN: DIAGRAMAS DE INTERACCIÓN N-M}

Si se considera que la sección está totalmente definida, i.e. se conocen las dimensiones de la sección transversal, las áreas de armadura $\left(A_{s}^{\prime}\right.$ y $\left.A_{s}\right)$ y sus posiciones $(d$ y $d$ ); las incógnitas en las ecuaciones de equilibrio [7] serán: $x, N_{u}(\mathrm{x})$ y $M_{u}(\mathrm{x})$.

Al diagrama definido por los pares $N_{u}(x)$ $M_{u}(x)$, obtenidos a partir de las ecuaciones [7], se le conoce como diagrama de interacción Axil-Flector (N-M).

Ejemplo. Sea una sección rectangular de $0,5 \times 0,5 \mathrm{~m}$ de hormigón $\mathrm{C}-30$ y acero B-500, $\operatorname{con} A^{\prime}=1571 \mathrm{~mm}^{2}$ (5Ø20), $A_{\mathrm{s}}=2454$ $\mathrm{mm}^{2}$ (5Ø25) con un recubrimiento mecánico de $50 \mathrm{~mm}$ (Figura 5). El diagrama de interacción se puede obtener directamente a partir de la expresión paramétrica dada por la expresión [7]; este diagrama se ha representado gráficamente en la Figura 5.

\section{EL PROBLEMA DE DISEÑO: DIAGRA- MAS DE ARMADO A FLEXIÓN RSD}

El problema de diseño de secciones de hormigón armado es más complejo dado que en ese caso las área de armadura, $A_{s}$ y $A_{s^{\prime}}^{\prime}$ son incógnitas del problema. Por el contrario, las acciones o solicitaciones de diseño, $N_{d}$ y $M_{d^{\prime}}$ son datos del problema. Si el diseño se realiza en rotura se habrá de verificar que: $N_{u}(\mathrm{x})=N_{d}$ y $M_{u}(\mathrm{x})=\mathrm{M}_{\mathrm{d}}$.

En este caso se trata de determinar las áreas de armadura necesarias, $A_{s}$ y $A_{s^{\prime}}^{\prime}$ para que la sección transversal de hormigón resista un par de diseño $N_{d}-M_{d}$. En este problema las incógnitas son tres $\left(A_{s^{\prime}} A_{s}^{\prime}\right.$ y $\left.x\right)$ y dos las ecuaciones (ecuaciones de equilibrio [7]). Por tanto, el problema de diseño en rotura de secciones transversales de hormigón tiene infinitas soluciones.

Por simplicidad se planteará el equilibrio tomando momentos a nivel del c.d.g. de

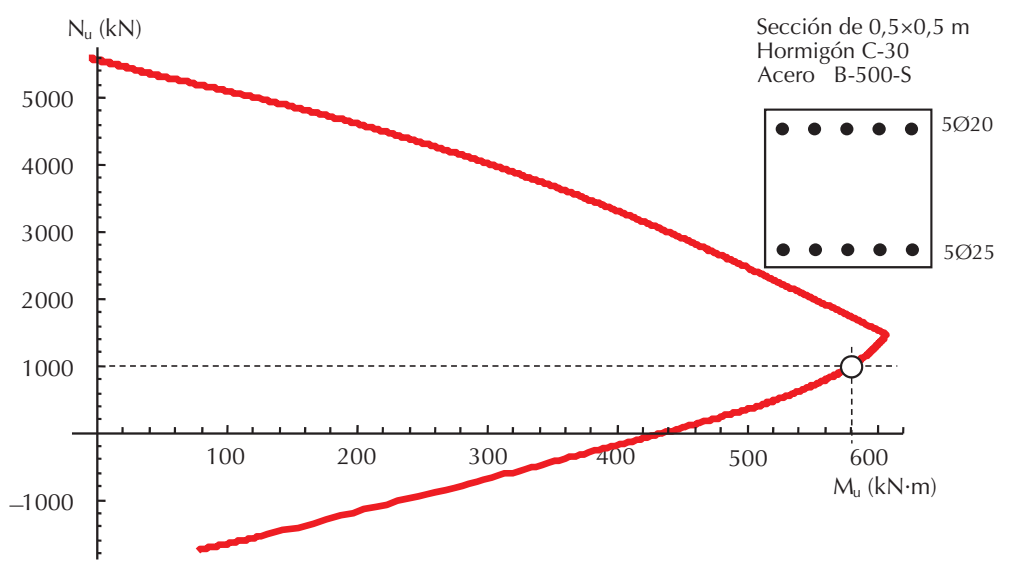

las armaduras superior e inferior (Figura 6). De este modo las expresiones resultantes se presentan desacopladas en $A_{s}$ y $A_{s^{\prime}}^{\prime}$ que en este caso serán funciones de $\mathrm{x}$ : [9]

[9]

5. Diagrama de interacción N-M

6. Equilibro en el cdg de la armadura inferior y superior

$$
\begin{aligned}
& A_{s}^{\prime}=A_{s}^{\prime}(x)=\frac{M_{d}-N_{d}\left(\frac{h}{2}-d\right)+N_{c}(x)\left(z_{c}(x)-d\right)}{\sigma_{s}^{\prime}(x)\left(d-d^{\prime}\right)} \\
& A_{s}=A_{s}(x)=\frac{M_{d}-N_{d}\left(\frac{h}{2}-d^{\prime}\right)+N_{c}(x)\left(z_{c}(x)-d^{\prime}\right)}{-\sigma_{s}(x)\left(d-d^{\prime}\right)}
\end{aligned}
$$

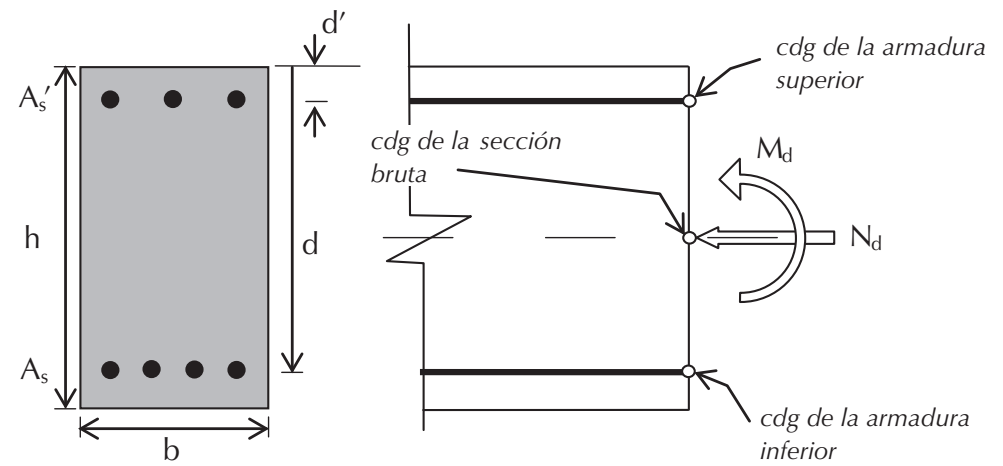

La representación gráfica de $\mathrm{A}_{s}(x), \mathrm{A}_{s}^{\prime}(x)$ y $\left(A_{s}(x)+A_{s}^{\prime}(x)\right)$ fue presentada por Hernández-Montes et ál. (6) bajo la denominación de RSD (del inglés Reinforcement Sizing Diagrams). La representación RSD permite visualizar las infinitas soluciones de $A_{s}(x)$ y $A_{s}^{\prime}(x)$ para las cuales una determinada sección transversal resiste -en rotura- unas solicitaciones $\mathrm{N}_{d}-\mathrm{M}_{d}$ dadas.

Es interesante conocer también la curvatura de rotura de la solución elegida, para ello se ha introducido un nuevo eje en el RSD de la Figura 7. Sin embargo debido al confinamiento proporcionado por la armadura transversal se pueden presentar curvaturas muy superiores a las indicadas en la Figura 7, ver Hernández-Montes et ál (7). La relación entre la profundidad de la fibra neutra $x$ y la curvatura de rotura $\varphi$ representada en la Figura 7 se obtiene mediante las ecuaciones [10] deducidas a partir de las ecuaciones [3] y de la Figura 4: 
7. Diagrama RSD de la sección del ejemplo, para $\mathrm{Nd}=1000 \mathrm{kN}$ y $\mathrm{Md}=580 \mathrm{kN} \bullet \mathrm{m}$

$$
\begin{gathered}
\varphi(x)=\left\{\begin{array}{lll}
\frac{\varepsilon_{c u 3}}{x} & \text { si } & 0 \leq x<h \\
\frac{\varepsilon_{c 3}}{x-\Xi} & \text { si } & x \geq h
\end{array}\right. \\
\text { donde } \Xi=h\left(1-\frac{\varepsilon_{c 3}}{\varepsilon_{c u 3}}\right)
\end{gathered}
$$

Consideremos el punto indicado en el diagrama de interacción de la Figura 5, correspondiente al par: $N_{d}=1000 \mathrm{kN}$ y $M_{d}=580 \mathrm{kN} \bullet \mathrm{m}$. A partir de las ecuaciones [9] se pueden obtener las áreas de armadura necesarias para que esta sección transversal de hormigón resista el par que la solicita. Las infinitas soluciones obtenidas de [9] son susceptibles de ser representadas en un diagrama RSD. Ver Figura 7.

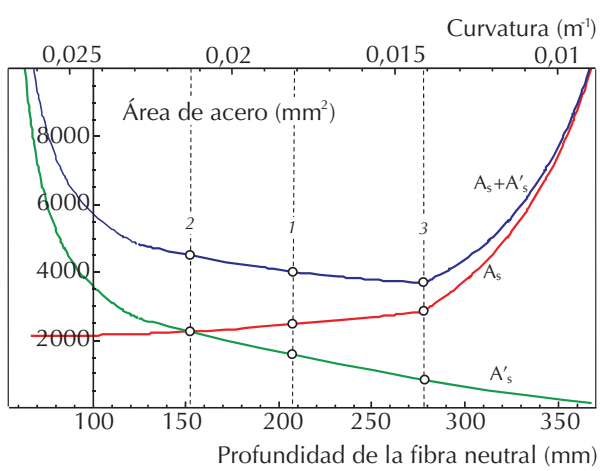

La línea vertical 1 de la Figura 7 corresponde a la solución del apartado anterior: $A^{\prime}=1571 \mathrm{~mm}^{2}$ (5Ø20), $A_{s}=2454 \mathrm{~mm}^{2}$ (5Ø25), para la cual la profundidad de la fibra neutra es $x=207,8 \mathrm{~mm}$. Otro caso particular de especial interés corresponde al armado simétrico $A_{s}^{\prime}=A_{s}=2251 \mathrm{~mm}^{2}$ (para $x=152,7 \mathrm{~mm}$ ), que se ha identificado con la línea vertical 2 en la Figura 7. Sin embargo, tal y como se puede apreciar en la Figura 7, ninguna de los dos casos particulares anteriores corresponde al mínimo de armadura total -línea vertical 3 de la Figura 7-, que se presenta para: $A_{s}^{\prime}=857 \mathrm{~mm}^{2}$ y $A_{s}=2834 \mathrm{~mm}^{2}(\operatorname{con} x=275,6 \mathrm{~mm})$.

y momento límite asociado:

Como ya deduce de la Figura 3

$$
x_{\text {lim }}=\frac{d}{1+f_{y d} / \varepsilon_{c u 2} E_{s}}
$$

De la Figura 3 se desprende que si $x>x_{\lim }$ el acero en tracción abandona la zona plástica y comienza a trabajar en la zona elástica, a una tensión inferior a $f_{y d}$. Por tanto, que si $x>x$ el acero está "desaprovechado". El momento generado por las tensiones internas para el valor $x=x_{\text {tim }}$ cuando no se dispone armadura de compresión $\left(A_{s}^{\prime}=0\right)$ se denomina $M_{\text {lim }}$.

2 Puede ser interesante comparar la mínima obtenida con la solución simétrica

En este caso la solución de área mínima -solución 3- también es de curvatura mínima, comparada con las soluciones 1 y 2 de la Figura 7. Esto no siempre es así como se puede ver en Hernández-Montes et ál. (7) principalmente porque las curvaturas últimas dependen fundamentalmente de la armadura transversal y para el cálculo de estas curvaturas es necesario utilizar modelos de arcos de descarga, ver (8).

\section{EL MÍNIMO DE ARMADURA TOTAL: EL TEOREMA DE ARMADO A FLEXIÓN}

Hernández-Montes et ál. (9) demostraron que, si se aplica el Eurocódigo 2, existe una caracterización para el mínimo de armadura total $\left(A^{\prime} s+A_{s}\right)$. Esta caracterización se recogió en el Teorema de Armado a Flexión ó TOSR (Theorem of optimal section reinforcement). Posteriormente, Gil-Martín et al. (10) demostraron que el teorema también se verificaba si se adoptan las hipótesis de la norma americana ACl-318.

\section{Teorema:}

En el caso de secciones transversales rectangulares con dos niveles de armadura se verifica que, de entre las infinitas posibilidades de armar la sección de hormigón en rotura, el mínimo total de armadura $\left(A_{s}+A_{s}^{\prime}\right)$ corresponde a una de las siguientes soluciones:

$-A_{s}=0 \quad y / 0 \quad A_{s}^{\prime}=0$

- $x$ igual o ligeramente superior a $x_{\text {lim }}\left(x_{\text {lim }}\right.$ está definido en la Figura 3)

- deformación constante en toda la sección transversal igual a $\varepsilon_{\text {ud }}$ (ver Figura 3)

- deformación constante en toda la sección transversal igual a $\varepsilon_{\mathrm{c} 2}-{ } \varepsilon_{\mathrm{c} 3}-$ (ver Figura 3).

\section{Corolario}

Como corolario del teorema anterior se concluye que el dimensionamento en rotura de secciones rectangulares de hormigón armado con dos niveles de armadura puede abordarse mediante alguna de las siguientes formas:

1. Armadura simétrica $\left(A_{s}=A_{s}^{\prime}\right)$. Esta solución es adecuada cuando el elemento estructural debe resistir distintos tipos de cargas (pares $N-M$ ) y el conjunto de todos los pares es relativamente simétrico respecto al eje de la $N$. Un ejemplo típico corresponde al diseño de columnas, en las que, debido al viento o al sismo, el máximo momento positivo y el mínimo negativo tienen valores absolutos muy parecidos.

2. Imponer que $A_{s}^{\prime}=0$. Esta solución es adecuada en vigas, siempre que el momento no sea superior a un momento denominado $M_{\text {lim }}{ }^{1}$.
3. Fijar el valor de una de las dos armaduras $A_{s}$ o $A_{s}^{\prime}$ o una relación entre ambas (i.e. $A_{s} / A_{s}^{\prime}=c t e$ ). En este caso el número de incógnitas se reduce a dos y el problema tiene solución única.

4. Imponer $x=x_{\text {lim }}$.

5. Calcular las armaduras superior e infema y elegir la solución de mínima armadura total ${ }^{2}$.

6. Realizar un diagrama RSD de armado a flexión y elegir la combinación de armaduras que más interese para cada caso concreto. rior para los cinco supuestos del teore- 


\section{DIAGRAMAS RSD PARA FLEXIÓN BIAXIAL}

El concepto de RSD se ha extendido al caso de flexión biaxial. Los resultados obtenidos han sido muy interesantes desde el punto de vista de su aplicabilidad, habiéndose obtenido un ahorro de acero que nunca antes había sido considerado (11).

Puesto que los modelos de acero y hormigón no dependen del tipo de flexión (uniaxial o biaxial) que se considere, las diferencias fundamentales entre flexión uniaxial y biaxial son:

- En el caso biaxial aparece una nueva ecuación de equilibrio asociada a la flexión en la otra dirección. En este caso son tres las ecuaciones de equilibrio: una de equilibrio de esfuerzos axiles y dos de equilibrio de momentos, ver (8).

- La posición de la fibra neutra en flexión uniaxial se define a partir de una sola variable $(x)$ mientras que en flexión biaxial se requieren dos variables para posicionar la fibra neutra: $\xi$ y $\varphi$ (Figura 8).

- Generalmente no bastará con disponer dos niveles de armadura y, de hecho, en secciones rectangulares es habitual disponer armadura en las cuatro caras.

Igual que para flexión uniaxial, para la comprobación de secciones transversales sometidas a flexión biaxial (i.e. sección totalmente definida) es habitual recurrir a los diagramas de interacción $N-M_{x-} M_{y}$. La sección podrá resistir los esfuerzos que la solicitan si la terna $\left(N_{d^{\prime}} M_{x d}\right.$ y $\left.M_{y d}\right)$ queda dentro del diagrama; en caso contrario la sección agotará.

Para el dimensionamiento de secciones sometidas a flexión biaxial tradicionalmente

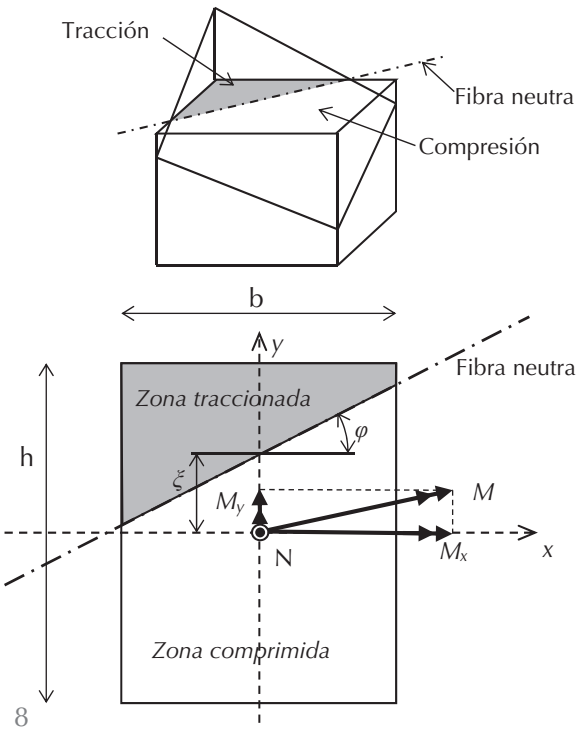

se forzaba a que el número de incógnitas fuera igual al número de ecuaciones de equilibrio. Para lograrlo, ha sido habitual en la práctica fijar la posición de las armaduras y emplear un único diámetro $\varnothing$ para todas las barras. De esta manera las variables o incógnitas se reducen a tres: $\xi, \varphi$ y $\varnothing$. El Prontuario informático del Hormigón 3.0 del IECA (12) utiliza este procedimiento.

Como se ha comentado, las soluciones típicas de armado en flexión biaxial consisten en barras de un mismo diámetro situadas a separación constante a lo largo del perímetro de la sección transversal. El diagrama RSD-Biaxial (8) (11) se plantea como una forma alternativa de dimensionamiento que puede llevar a ahorros importantes de armadura.

Si se consideran dos separaciones distintas e iguales en las caras paralelas $\left(s_{v}\right.$ y $s_{h^{\prime}}$ ver Figura 9) con objeto de conservar la simetría de la armadura, el número de incógnitas aumenta a cuatro: $\xi, \varphi, \varnothing$ y $s_{h} / s_{v}$. En esta situación, las tres ecuaciones de equilibrio se pueden expresar como: [11]

$$
\begin{aligned}
& N=\sum N\left(\xi, \varphi, \varphi, s_{h} / s_{v}\right) \\
& M_{x}=\sum M_{x}\left(\xi, \varphi, \varphi, s_{h} / s_{v}\right) \\
& M_{y}=\sum M_{y}\left(\xi, \varphi, \varphi, s_{h} / s_{v}\right)
\end{aligned}
$$

En el caso de dimensionamiento en rotura en la situación de flexión uniaxial, como hemos visto antes, existen infinitas soluciones de armado. Análogamente, en flexión biaxial considerando dos separaciones distintas, $s_{h}$ y $s_{y^{\prime}}$ las ecuaciones de equilibrio son 3 y las incógnitas 4 por lo que existen también infinitas soluciones. En ambos casos (flexión uniaxial y flexión biaxial con dos separaciones iguales en caras paralelas) el número de incógnitas es sólo uno mayor que el de ecuaciones y por lo tanto resolu-

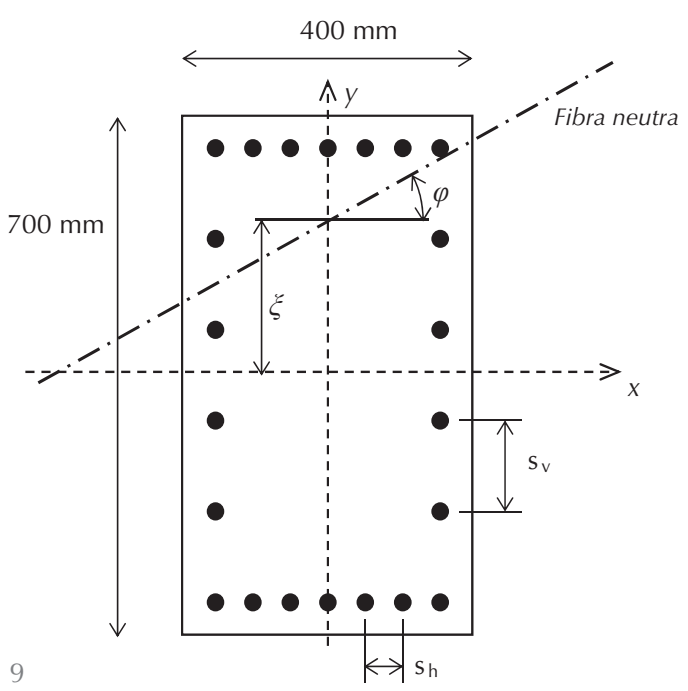

8. Posición de la fibra neutra en flexión biaxial.

9. Ejemplo de Diagrama RSDBiaxial. $\mathrm{f}_{\mathrm{ck}}=30 \mathrm{MPa} ; \mathrm{f}_{\mathrm{yk}}=500 \mathrm{MPa}$

recubrimiento mecánico $=7 \mathrm{~mm}$

$$
\text { Caso } 1 \text { Caso } 2
$$

$\mathrm{N}_{\mathrm{d}}=200 \mathrm{kN} 200 \mathrm{kN}$

$M_{x d}=300 \mathrm{kN} \cdot \mathrm{m} 400 \mathrm{kN} \cdot \mathrm{m}$

$M_{y d}=250 \mathrm{kN} \cdot \mathrm{m} 100 \mathrm{kN} \cdot \mathrm{m}$ 


\section{Diagramas RSD-biaxiales}

11. Planteamiento general para el diseño en rotura de elementos de hormigón armado sometidos a flexión.

12. Pilote asimétrico hormigonado. ble en función de una sola variable y, por tanto, representable gráficamente en 2D. En el caso de la flexión biaxial se ha decidido utilizar como variable -eje de abscisas- el área de la armadura situada en la parte superior más la de la situada en la parte inferior mientras que en el eje de ordenadas se
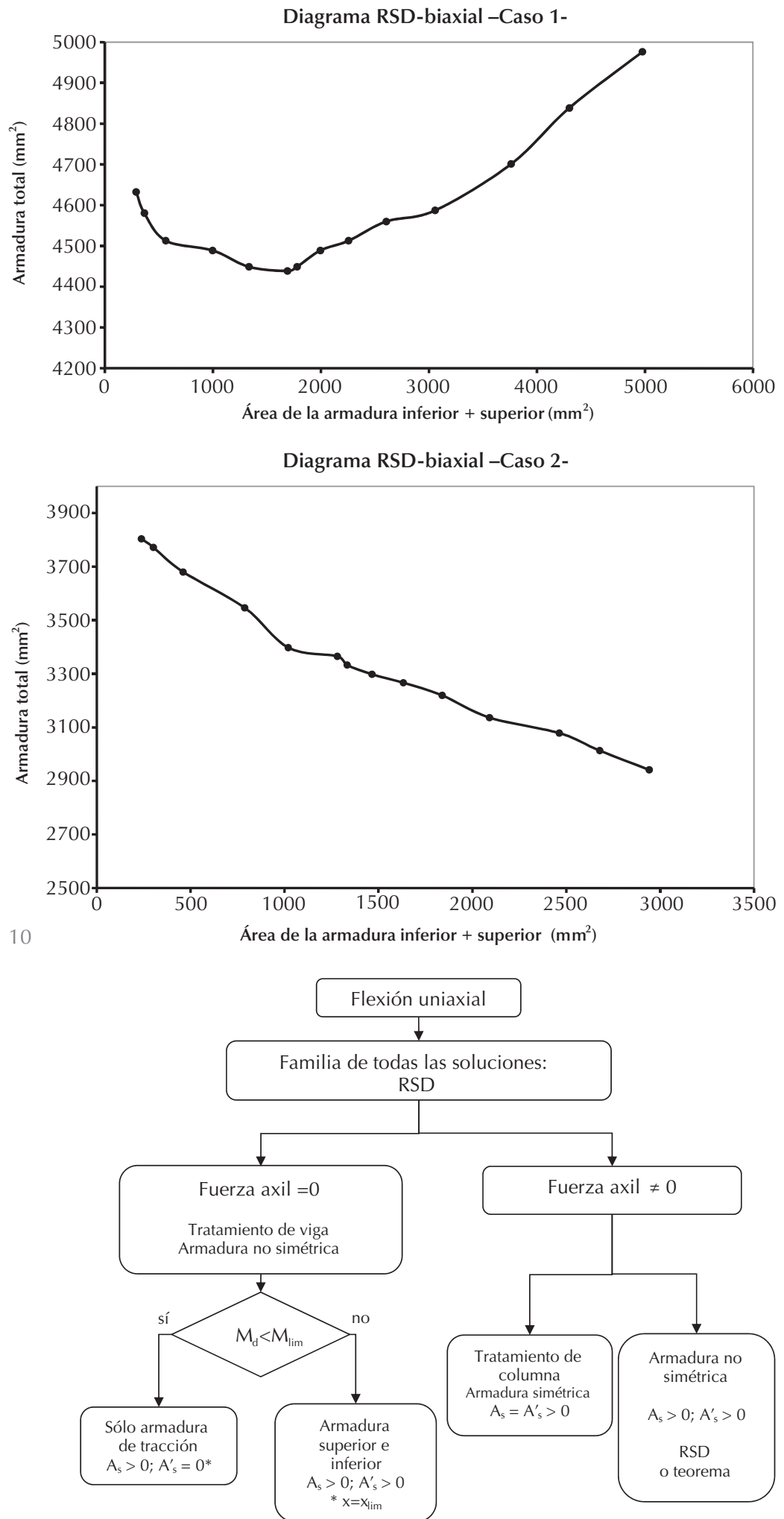

* El teorema de armado a flexión garantiza que, para secciones rectangulares, este caso corresponde a la mínima cuantía.

ha representado el área de armadura total. Esta elección permite localizar la existencia del mínimo de armadura.

En la Figura 10 se han representado los diagramas RSD-biaxial correspondientes a los dos casos prácticos especificados en la Figura 9. Como se puede apreciar, estas representaciones permiten obtener la solución óptima -o de mínimo armado- de manera inmediata. Las irregularidades en las gráficas se deben fundamentalmente al cambio brusco de ancho en función del valor de $\varphi$ (ver Figura 9).

\section{CONCLUSIONES}

Las bases para la comprobación y el diseño en rotura de elementos de hormigón armado y pretensado fueron ya establecidas en la década de los años 50 del siglo pasado. Al igual que en otras muchas disciplinas algunas de estas aportaciones siguen siendo aún útiles desde el punto de vista de su aplicación práctica, como es el caso de los diagramas de interacción N-M. Sin embargo existen desarrollos que, aunque en su época supusieron un avance importante en esta disciplina, en la actualidad su utilidad puede y deber ser cuestionada.

El desarrollo de la matemática y los grandes avances que el cálculo numérico ha conseguido en las últimas décadas han dejado obsoletas algunas de las hipótesis que tradicionalmente se han venido considerando para dimensionar secciones de hormigón armado en rotura. En la actualidad, las potentes herramientas de las que dispone el ingeniero le permiten realizar un diseño más racional y económico de los elementos de hormigón armado sin necesidad de adoptar, sin más, los planteamientos tradicionales. Concretamente, en la actualidad no es necesario proceder a la división entre grandes y pequeñas excentricidades, como se ha venido haciendo anteriormente.

La Figura 11 plantea una manera compacta $y$ totalmente novedosa de plantear el

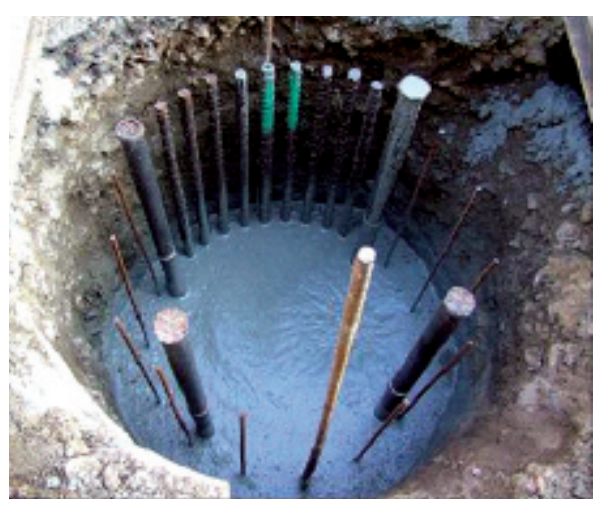


dimensionamiento de secciones de hormigón armado, basada en los Diagramas de Armado a Flexión (RSD) y en el Teorema de Armado a Flexión (TORS).

Sólo un planteamiento compacto como el planteado por las ecuaciones [3 a 6] permite llevar a cabo una optimización de la sección transversal. El procedimiento de optimización de la armadura longitudinal se ha aplicado a los "pilotes asimétricos", patentados por la Universidad de Granada, que han sido recientemente puestos en obra. Ver Figura 12.
En el caso de flexión biaxial, los diagramas RSD también han demostrado ser una herramienta idónea para realizar un diseño más racional y económicamente más atractivo que los planteamientos tradicionales.

\section{AGRADECIMIENTOS}

Los autores desean expresamente agradecer la contribución anónima de los dos revisores de este artículo, su tiempo y dedicación han ayudado a mejorar la configuración final del trabajo.

\section{BIBLIOGRAFÍA}

(1) Whitney, C.S.; Cohen, E.: "Guide for Ultimate Strength Design of Reinforced Concrete". ACl Journal, Vol. 28, № 5, (1956). (Proceedings V.53), pp. 445-490.

(2) García Meseguer, A.; Morán Cabré F.; Arroyo Portero, J.C.: Jiménez Montoya. Hormigón Armado. p. 660, Editorial Gustavo Gili, Barcelona, 2009.

(3) Walther, R. ; Miehlbardt, M.: Dimensionnement des structures en Béton. p.388, Press Polytechniques et Universitaires Romandes, Lausanne, 1990.

(4) Páez, A.: Hormigón Armado (Tomo 1). p. 635, Editorial Reberté, Barcelona, 1986.

(5) Gil-Martín, L.M.; Hernández-Montes, E.; Aschheim, M.; Pantazopoulou. S.J.: "Aproxímate expressions for the simulated response of slender longitudinal reinforcement in monotonic compression". Magazine of Concrete Research, Vol. 60 (2008), pp. 391-397.

(6) Hernández-Montes, E.; Gil-Martín, L.M.; Aschheim M.: "Design of concrete members subjected to uniaxial bending and compression using reinforcement sizing diagrams". ACI Structural Journal, Vol. Enero-Febrero (2005), pp.150-158.

(7) Hernández-Montes, E., Aschheim, M and Gil-Martín, L.M. (2004). "The impact of optimal longitudinal reinforcement on the curvature ductility capacity of reinforced concrete column sections". Magazine of Concrete Research, Vol. 56, № 9, pp. 499-512.

(8) Hernández-Montes, E.; Gil-Martín L.M.: Hormigón Armado y Pretensado. Concreto reforzado y preesforzado. Editorial Ingeniería e Infraestructuras. Granada, 2007. http:// www.ugr.es/ emontes/prensa/HormigonEstructural.pdf

(9) Hernández-Montes, E.; Gil-Martín, L.M.; Pasadas-Fernández, M.; Aschheim M.: "Theorem of optimal reinforcement for reinforced concrete sections". Structural and Multidisciplinary Optimization. Vol 36 (2008), pp. 509-521.

(10) Gil-Martín, L.M.; Aschheim, M.; Hernández-Montes, E.; Pasadas-Fernández. M.: "Recent developments in optimal reinforcement of RC beam and column sections". Engineering Structures, Vol. 33 nº (2011), pp. 1170-1180.

(11) Gil-Martín, L.M.; Hernández-Montes, E.; Aschheim, M.:“Optimal Reinforcement of RC columns for biaxial bending". Materials and Structures, Vol. 43 n9 (2010), pp. 12451256.

(12) Corres Peiretti, H.; Martínez Martínez, J.L.; Pérez Caldentey A.; López Agüí J.C. Prontuario Informático del Hormigón Estructural 3.0. IECA. (www.ieca.es). 